\title{
Le sous-financement gouvernemental et son impact sur le développement des asiles francophones au Québec (1845-1918)
}

\author{
André M. Paradis
}

Volume 50, numéro 4, printemps 1997

URI : https://id.erudit.org/iderudit/305602ar

DOI : https://doi.org/10.7202/305602ar

Aller au sommaire du numéro

Éditeur(s)

Institut d'histoire de l'Amérique française

ISSN

0035-2357 (imprimé)

1492-1383 (numérique)

Découvrir la revue

Citer cet article

Paradis, A. M. (1997). Le sous-financement gouvernemental et son impact sur le développement des asiles francophones au Québec (1845-1918). Revue d'histoire de l'Amérique française, 50(4), 571-598.

https://doi.org/10.7202/305602ar
Résumé de l'article

Cet article s'intéresse à l'impact du sous-financement gouvernemental sur le développement des établissements asilaires au Québec entre 1845 et 1918. Dès le départ, la préférence accordée par les hommes politiques au système d'affermage, lequel permet au gouvernement de maintenir ses dépenses à un très bas niveau, exerce des effets délétères sur la qualité des soins et le développement de la psychiatrie. Déjà l'un des plus bas en Amérique du Nord, le financement des asiles québécois chute dramatiquement après 1871, au fur et à mesure que la population des aliénés internés augmente et que les besoins deviennent plus criants. La loi de 1885 et l'enquête de 1887 sur les asiles ébranlent pourtant le système d'affermage et ouvrent la porte à une lente modernisation des institutions asilaires. La période de 1890 à 1918 s'avère en effet une période de transition entre l'asile-prison et l'asile-hôpital; si les problèmes financiers et, conséquemment, les problèmes d'aménagement de l'espace asilaire restent aigus, l'amélioration de la progression des soins annonce les gains ultérieurs de la psychiatrie.
Tous droits réservés @ Institut d'histoire de l'Amérique française, 1997

Ce document est protégé par la loi sur le droit d'auteur. L'utilisation des services d'Érudit (y compris la reproduction) est assujettie à sa politique d'utilisation que vous pouvez consulter en ligne.

https://apropos.erudit.org/fr/usagers/politique-dutilisation/ 


\title{
LE SOUS-FINANCEMENT GOUVERNEMENTAL ET SON IMPACT SUR LE DÉVELOPPEMENT DES ASILES FRANCOPHONES AU QUÉBEC (1845-1918)
}

\author{
ANDRÉ M. PARADIS* \\ Centre interuniversitaire d'études québécoises \\ Université du Québec à Trois-Rivières
}

\section{RÉSUMÉ}

Cet article s'intéresse à l'impact du sous-financement gouvernemental sur le développement des établissements asilaires au Québec entre 1845 et 1918. Dès le départ, la préférence accordée par les hommes politiques au système d'affermage, lequel permet au gouvernement de maintenir ses dépenses à un très bas niveau, exerce des effets délétères sur la qualité des soins et le développement de la psychiatrie. Déjà l'un des plus bas en Amérique du Nord, le financement des asiles québécois chute dramatiquement après 1871 , au fur et à mesure que la population des aliénés internés augmente et que les besoins deviennent plus criants. La loi de 1885 et l'enquête de 1887 sur les asiles ébranlent pourtant le système d'affermage et ouvrent la porte à une lente modernisation des institutions asilaires. La période de 1890 à 1918 s'avère en effet une période de transition entre l'asile-prison et l'asile-hôpital: si les problèmes financiers et, conséquemment, les problèmes d'aménagement de l'espace asilaire restent aigus, l'amélioration de la progression des soins annonce les gains ultérieurs de la psychiatrie.

\section{ABSTRACT}

This article deals with the impact of government under-financing upon the development of asylums in Québec between 1845 and 1918. From the outset, politicians preferred the system of sub-contracting because it kept the costs down; such a choice, however, had deleterious effects upon the level of care and the development of psychiatry. Already one of the lowest in North America, the financing of Québec asylums decreased after 1871 as more insane patients were interned and the needs became ever more crying. The 1885 law and the 1887 inquiry on asylums called into question the practice of sub-contracting and launched a gradual movement of modernization of asylums. The period 1890 to 1918 witnessed a transition from the concept of the asylum as prison to that of asylums as hospital: financial difficulties and space problems remained severe, but gradual improvement in care led to later progress of psychiatry.

* André M. Paradis est décédé en janvier 1997. Le Comité de rédaction souhaite rendre hommage à ce collègue et à son importante contribution à la recherche en publiant cet article dont l'auteur n'a malheureusement pas pu voir la dernière version. Nous remercions madame Thérèse Paradis qui nous a accordé l'autorisation de publier, ainsi que monsieur François Guérard pour sa collaboration à la préparation du manuscrit.

RHAF, vol. 50, $\mathrm{n}^{\circ} 4$, printemps 1997

[571] 


\section{INTRODUCTION}

Il existe un consensus quasi universel qui veut que le système asilaire, inauguré par Tuke en Angleterre et par Pinel en France, ne put jamais répondre aux espoirs qu'y avaient déposés les pères fondateurs de la psychiatrie, et que l'entreprise se solda presque partout en Occident par une déconvenue. On avait en effet pensé pouvoir pratiquer dans les asiles cette fine fleur de la philosophie des Lumières et de l'idéologie qu'on appelait à l'époque le traitement moral. L'asile devait permettre en principe d'arracher les fous à leurs cachots, de les soustraire à toute décision juridique arbitraire, de les libérer des stimulations pathogènes de leur milieu et de les protéger contre toute violence gratuite. Il devait les rééduquer aux bonnes manières et leur redonner le goût d'une existence saine et laborieuse en attirant leur attention sur de nouvelles idées et de nouveaux plaisirs. Mieux, l'asile devait permettre d'étudier la folie sous tous ses angles et de faire progresser la médecine autant que la science de l'esprit humain. Dans l'esprit des fondateurs de la psychiatrie, il était inévitable qu'une telle entreprise eût des retombées considérables sur la société tout entière, car, connaissant mieux les causes et les effets des maladies de l'esprit, on pourrait non seulement les prévenir mais miser aussi sur les leçons de l'expérience pour élaborer le programme d'une éducation éclairée.

L'intention de Tuke et de Pinel était noble et on ne peut certainement pas leur reprocher d'avoir cru en la grandeur de leur projet. Tout compte fait, on peut dire que l'institution asilaire servit effectivement à faire progresser notre connaissance des symptômes de la folie. Avec le temps, elle finit même par rendre caducs de nombreux mythes: ceux, entre autres, de la déraison, de l'incurabilité et de la violence intrinsèque des aliénés. L'asile permit aussi d'humaniser le régime qu'on leur réservait ailleurs dans un anonymat beaucoup plus redoutable. Mais, hélas, l'asile ne fut pas pour autant le fruit d'un plan d'action et de connaissance raisonné. Il faut se rappeler, en effet, que l'institution asilaire vint à l'heure où le cumul des frustrations engendrées par la rapacité du gain, par la concurrence effrénée, par les conditions de travail souvent inhumaines, par les migrations massives de population et par l'incertitude des lendemains mettait à rude épreuve l'équilibre mental des collectivités. Elle vint à l'heure où l'ampleur de la pauvreté et de la misère en milieu urbain exigeait qu'on repense de fond en comble la gestion des ressources consacrées au contrôle des destitués: mendiants, itinérants, criminels, alcooliques,

1. Cet article a été rendu possible grâce au soutien de la bibliothèque Osler et grâce au Conseil de recherches en sciences humaines du Canada. 
syphilitiques, prostitués, toxicomanes, déficients intellectuels, épileptiques et impotents, devenus invivables et trop lourds à porter pour leur famille, ou jugés dangereux pour leur entourage. L'asile vint donc à l'heure où on dut le considérer d'abord et avant tout non pas comme une institution médicale, mais comme une entreprise de charité et de sécurité publiques, voire comme un prolongement de l'institution carcérale, les prisons lui fournissant au milieu du siècle dernier plus de la moitié de sa clientèle. Mais avec l'essor de la société industrielle, personne n'avait prévu à quel point l'institution asilaire était promise à la prolifération et au gigantisme.

À compter de 1850, l'asile devint un rouage essentiel dans l'effort de normalisation sociale et une composante incontournable de l'économie politique. En Allemagne, par exemple, le nombre des aliénés internés, qui n'était que de 200 en 1801, grimpa à 45000 en fin de siècle. Le nombre des établissements asilaires passa alors de 3 à $55^{2}$. En Angleterre, la population asilaire se multiplia par treize entre 1807 et $1890^{3}$, tandis qu'aux États-Unis la croissance démographique des asiles prit des proportions encore plus accentuées: le nombre des patients internés, qui n'était que de 2561 en 1844, grimpa à 40942 en 1880 , puis à 74028 en $1890^{4}$. Et là encore, selon l'historien Grob, l'asile était loin de répondre adéquatement à la demande; les aliénés internés ne représentaient, en effet, qu'une petite fraction des malades mentaux identifiés comme tels dans la population: au plus $25 \%$, nous assure Grob ${ }^{5}$. C'est dire à quel point l'Etat, en Europe comme ici, accusait du retard sur le développement global de la société et à quel point l'internement des aliénés devait peser sur les finances publiques.

Presque tous les établissements asilaires en Amérique du Nord durent très tôt apprendre à vivre avec les contractions budgétaires et les aléas de la charité publique. Mais il y a peu d'endroits où la carence en fonds et, par conséquent, la carence en ressources matérielles et cliniques, furent aussi durement et aussi longtemps ressenties

2. Cf. Thomas Höll et Paul-Otto Schmidt-Michel, Irrenpflege im 19. Jahrhundert (Band AA, 1989), 16-17.

3. Marlene Arieno, Victorian Lunatics: a Social Epidemiology of Mental Illness in MidNineteenth Century England (University Microfilm International, Dissertation Information Service, 1984), vi.

4. S. W. Hamilton, «The History of American Mental Hospitals», dans J. K. Hall, One Hundred Years of American Psychiatry (New York, Columbia University Press, 1944), 86 et 112. (Angleterre/Galles, 1844: 20 983; Écosse, 1844: 4 500; France, 1841: 19 738; États-Unis, 1840: 17 434).

5. Cf. G. N. Grob, Mental Illness and American Society (1875-1945) (Princeton, Princeton University Press, 1983), 8. On trouvera d'autres données statistiques dans l'excellent volume de Charles E. Goshen, comp., Documentary History of Psychiatry; a Source Book on Historical Principles (New York, Philosophical Library, 1967), 387-390. 
qu'au Québec. Non seulement le financement gouvernemental des asiles québécois au $\mathrm{XIX}^{\mathrm{e}}$ siècle compta au départ parmi les plus bas en Amérique du Nord ${ }^{6}$, mais il chuta de façon draconienne au fur et à mesure que la population des aliénés internés augmentait et que les besoins en infrastructures et en personnel devenaient plus criants. On peut, bien sûr, imputer le fléchissement des allocations du gouvernement à la longue crise économique qui sévit entre 1870 et 1900 . Et on comprend qu'avec de petits moyens l'État ait été tenté de relancer la formule d'affermage en faisant appel à la collaboration de particuliers ou de corporations religieuses. Mais la conjoncture économique ne peut expliquer à elle seule que les subventions consenties aux asiles par le gouvernement aient été de cinq à six fois inférieures à celles de la province d'Ontario ${ }^{7}$ ou à celles des États américains. En d'autres termes, le désengagement de l'État ne peut s'expliquer que parce que les partis au pouvoir en ont fait un atout majeur de leur politique et parce que les propriétaires d'asiles, d'une manière ou d'une autre, y ont aussi trouvé leur compte. L'empressement du haut clergé à s'opposer à la loi Ross de $1885^{8}$ illustre bien ce que pouvait représenter la propriété privée des asiles pour l'Église, surtout à une époque où culminait l'idéologie ultramontaine. Quant aux premiers propriétaires de Beauport, qu'il s'agisse du docteur Douglas ou du docteur Landry, on prétend qu'ils firent fortune avec leur entreprise. Compte tenu de la minceur de leurs revenus, on peut se demander comment cela a pu être possible, tout comme on peut se demander dans quelle mesure les propriétaires d'asiles ne devinrent pas, avec le changement d'échelle de la population asilaire, captifs d'un système de plus en plus lourd à gérer, de moins en moins lucratif, et dont la visée essentielle était de décharger l'État d'engagements trop onéreux.

J'ai tenté de démontrer dans un article récent ${ }^{9}$, sur la base des dépositions de la Commission royale d'enquête de 1887-1888, que le

6. Aux États-Unis, vers 1890 , le budget de fonctionnement versé per capita se situait entre 215 et 260\$: ainsi, par exemple, pour le North Dakota Hospital for Insane: 256,78\$; le Morristown State Asylum au New Jersey: 233,28\$; le Government Hospital for the Insane de Washington: $220 \$$ et les 9 asiles de l'État de New York: 217,85\$.

7. Dans leur lettre à Chauveau, datée du 15 juillet 1871 (voir à ce propos les Documents de la session, $\mathrm{n}^{\circ} 17$ (1871): 11), les propriétaires de Beauport, F.-E. Roy et J.-E. Landry, faisaient remarquer au secrétaire de la province que les seuls bâtiments de l'asile de Toronto avaient coûté au trésor du Haut-Canada la somme faramineuse de 600000 à $700000 \$$. C'était à l'époque l'équivalent d'au moins cinq années d'aide financière gouvernementale.

8. Cette loi qui limitait les droits de gérance des propriétaires et qui ordonnait le transfert de la responsabilité des soins à un bureau médical nommé par le gouvernement causa tout un émoi à l'époque. Farouchement contestée aussi par les propriétaires, elle aboutit à la création de la Commission royale d'enquête de 1887-1888.

9. «L'asile québécois et les obstacles à la médicalisation de la folie (1845-1890)», Bulletin canadien d'histoire de la médecine/Canadian Bulletin for the History of Medicine, 11 (1994): 297-334. 
régime d'affermage eut des effets ruineux sur l'évolution des établissements asilaires avant 1890: il en eut sur la lenteur des propriétaires à acquérir des bâtiments adéquats, sur la surpopulation et les conditions d'hygiène flageolantes des espaces d'internement, sur le manque de classification des patients, sur les taux de mortalité particulièrement élevés, sur les conditions de travail déplorables des médecins internes, sur le nombre, la formation et la rémunération des gardiens, sur le recours excessif aux mesures de contention et, finalement, sur le traitement moral qui, jusqu'au milieu des années 1890, demeura à peu près lettre morte. Le but de cet article était de faire contrepoids à l'ouvrage de Peter Keating ${ }^{10}$ qui, avec Françoise Boudreau ${ }^{11}$, est un des rares auteurs à nous avoir proposé un point de vue «critique» sur l'histoire asilaire au Québec. Keating, incidemment, opte pour une lecture optimiste de cette histoire. Il postule non seulement que le traitement moral se pratiqua dès 1830 à l'Hôpital général de Québec, mais aussi que l'enseignement de la psychiatrie trouva très vite un terrain fertile dans nos institutions médicales. Or cet optimisme me paraît excessif. Non seulement pour des raisons techniques ${ }^{12}$, mais aussi parce qu'il escamote allègrement certains des témoignages les plus éloquents du $\mathrm{XIX}^{\mathrm{e}}$ siècle, en l'occurrence ceux des aliénistes Bucknill, Hurd et D. H. Tuke qui vinrent visiter les deux grands asiles du Québec entre 1876 et 1884 . Keating passe aussi sous silence les déclarations de T. J. W. Burgess qui, soit dit en passant, fut le premier historien contemporain de l'institution asilaire au Canada. Il ne tient pas compte non plus des dépositions du personnel médical de Beauport et de Saint-Jean-de-Dieu (Turcotte, Perreault, Marois, Vallée, Bélanger et Devlin), enregistrées par la Commission royale de 1887-1888. Or, tous ces témoignages sont loin de militer en faveur d'une vision optimiste. Ils montrent au contraire qu'entre la théorie et la pratique, l'écart peut être parfois immense.

Dans le présent article, je rappellerai brièvement en quoi consistait le système d'affermage et quels effets délétères il eut sur l'évolution de l'institution asilaire avant 1890. J'essaierai surtout de montrer en quoi ces effets persistèrent durant la période qui va de 1890 jusqu'à la fin de la Première Guerre mondiale. On peut qualifier cette période

10. Peter Keating, La science du mal. L'institution de la psychiatrie au Québec, 18001914 (Montréal, Boréal, 1993).

11. Françoise Boudreau, De l'asile à la santé mentale (Montréal, Éditions coopératives Albert Saint-Martin, 1984).

12. Il faudrait montrer, entre autres, que les conditions de réclusion dans les loges étaient compatibles avec le traitement moral et que celui-ci pouvait être poursuivi en l'absence d'un aliéniste et en marge du contexte idéologique qui fut celui de Pinel; il faudrait aussi montrer en quoi consistait l'enseignement spécialisé auquel fait allusion Keating. 
de période de transition. Pour employer une expression du docteur Brochu, elle marque en effet le passage de «l'asile-prison» à «l'asilehôpital». Cette période se caractérise par des difficultés considérables quant à l'aménagement de l'espace asilaire, mais aussi par des améliorations gagnées de haute lutte en ce qui a trait à la médicalisation des soins. J'ai pensé qu'il était important de les souligner parce que ce sont ces gains qui ont ouvert la porte aux progrès ultérieurs de la psychiatrie: après 1916, le traitement des traumatismes crâniens et des névroses de guerre, l'attention beaucoup plus systématique donnée à l'étude des maladies nerveuses ainsi que l'adoption de mesures de dépistage et de prévention en hygiène mentale (grâce, en l'occurrence à l'utilisation des tests psychologiques en milieu scolaire) ont considérablement relevé le profil et la visibilité de cette discipline, même si la prévalence quasi dogmatique accordée à la théorie de la dégénérescence a beaucoup limité sa capacité d'innover.

\section{LA SITUATION ADMINISTRATIVE ET FINANCIÈRE DES ASILES AVANT 1890}

Le premier obstacle au développement des établissements asilaires au Québec fut sans contredit la préférence donnée au départ au système d'affermage. Ce système, contrairement au système de l'asile d'État en vigueur dans la très grande majorité des établissements canadiens, américains et européens, consistait à confier à contrat le soin et la garde des aliénés à des particuliers. Les avantages que le gouvernement retirait de ce système étaient nombreux: en échange d'une allocation annuelle dont le montant ne couvrait en principe que les frais d'entretien des patients, tels que stipulés aux contrats, le gouvernement n'avait à se soucier ni de l'achat ni de l'entretien des bâtiments d'asile, pas plus qu'il n'avait à se préoccuper de l'embauche, de la formation et des conditions de travail du personnel, qu'il soit médical ou de soutien. Il renonçait pratiquement à toute prétention en ce qui concerne la qualification, le rôle et le nombre de médecins traitants et confiait le traitement des malades au droit de gérance des propriétaires. Quant aux frais de couverture, essentiels pour ce genre d'entreprise particulièrement risqué, ils étaient aussi laissés à la charge des propriétaires. Paradoxalement, les inspecteurs d'asiles se félicitaient grandement à l'époque de ce genre d'arrangement qui avait pour effet de réduire à peu de chose la participation de l'État. C'était là une constante de leur discours. Plus sensibles aux économies réalisées qu'aux réalisations concrètes, ils écrivaient dans leur rapport pour l'année 1894: 
Si donc, comme le prétend le New York Medical World du mois de janvier 1895 la province d'Ontario est le porte-étendard des provinces du Dominion par l'excellence des établissements qu'elle consacre au traitement de ses aliénés, la province de Québec à coup sûr n'a pas à rougir de ses propres asiles [...] Ici: aucun édifice à construire, à réparer ou à entretenir, aucun ameublement ou article de literie à fournir, aucun employé à loger et à nourrir, aucun salaire à payer outre que ceux de six à sept médecins au montant de mille à dix-huit cents piastres par année [...] Dans la province de Québec, le système adopté pour la régie des asiles ne comportant aucun de ces grands déboursés, il s'en suit nécessairement que, tout en étant aussi efficace, quant aux guérisons, ces institutions soutirent du trésor provincial et des particuliers près de la moitié moins que les institutions similaires d'Ontario requièrent [en frais d'entretien pour les patients] ${ }^{13}$.

Le seul droit, inéluctable, que se réservait en fait le gouvernement, c'était de faire visiter les asiles de temps à autre par des inspecteurs et des médecins à sa solde. Les premiers, généralement plus soucieux de l'état des finances publiques que du bien-être des patients, veillaient à ce que chaque établissement s'acquitte le plus raisonnablement possible de ses engagements; les seconds devaient s'assurer que chaque cas d'internement et d'élargissement répondait bien aux dispositions de la loi.

C'est dire que, dans le système d'affermage, les propriétaires d'asiles avaient toute l'autorité, mais aussi toute la responsabilité, en ce qui concerne l'expansion et la modernisation de leurs établissements. C'est à eux, à leurs moyens et à leur discrétion, qu'incombait la décision d'ajuster leur investissement immobilier autant que leurs dépenses courantes à la demande. Cette situation, à peu près unique en Amérique du Nord, eut de nombreux inconvénients. Le plus important fut sans doute la tentation chronique des propriétaires de mieux asseoir leur profit en rognant systématiquement sur leurs dépenses, en particulier celles reliées à l'embauche du personnel médical, jugé onéreux et souvent même inutile, et du personnel des gardiens, généralement deux fois moins nombreux et deux fois moins bien rémunéré que celui des asiles de l'Ontario et des États-Unis ${ }^{14}$. Concrètement, cela signifie que les restrictions apportées aux dépenses de fonction-

13. Vingt-cinquième rapport des inspecteurs des prisons, asiles et bureaux publics de la province de Québec pour l'année 1894, Documents de la session, II,9 (1895): 108-109.

14. En 1888, par exemple, les gardiens de l'asile de Beauport étaient payés de 8 à $10 \$$ par mois en hiver et de $12 \$$ en été. Les gardiennes recevaient entre 4,50 et $5,50 \$$. À la même époque, à London et à Toronto, les gardiens gagnaient entre 18 et $26 \$$ et les gardiennes entre 10 et $12 \$$. Rapport de la commission des asiles d'aliénés, Documents de la session, 87 (1888): 28,117 et 122 . 
nement devaient inéluctablement se traduire par la lenteur de l'institution à se médicaliser, à développer une véritable expertise clinique et à promouvoir la psychiatrie comme profession. L'encadrement insuffisant des patients contraignit longtemps les médecins internes à recourir plus souvent qu'ils ne l'auraient voulu aux mesures agressives ou limitatives de contention. Le climat des établissements asilaires s'en trouva détérioré. L'application du traitement moral, qui supposait une politique d'organisation du travail et la mise sur pied d'activités de loisir, en souffrit lui aussi puisqu'il impliquait des dépenses d'infrastructures considérables, notamment pour l'organisation des ateliers. Les inspecteurs d'asiles, généralement très modérés et conservateurs dans leurs propos, étaient unanimes à dire dans les années 1880 que les aliénés n'avaient pratiquement pas d'occupations pour les distraire et que la plupart passaient leur temps à traîner passivement dans leurs quartiers.

Un autre inconvénient du système d'affermage fut de soustraire dans une bonne mesure les asiles à l'évaluation critique du public et à toute forme d'incursion gênante, d'en faire une chasse-gardée et de les couper de toute forme de participation communautaire vraiment active. Dans ce contexte d'anonymat, l'inspection des asiles prit ellemême rapidement l'allure d'une procédure purement formelle et la rédaction des rapports trimestriels des inspecteurs, celle d'un exercice sans lien avec la condition réelle des aliénés. Leurs rapports restèrent sur les tablettes des bureaux publics et aucun suivi ne fut donné à leurs recommandations. L'institution se perdit finalement dans une mer de mots. Il fallut attendre que la situation des aliénés internés soit jugée lamentable par la presse médicale et par des aliénistes étrangers en visite, tels Bucknill, Hurd et Tuke justement, pour que les rapports officiels des inspecteurs d'asiles cessent de se complaire dans un optimisme béat.

Mais le vice primordial du système d'affermage fut sans contredit la médiocrité même du financement de base des asiles et l'obligation de facto faite aux propriétaires et au personnel de multiplier les arrangements qui favorisaient la parcimonie. Avec un peu de recul, on constate en effet que le financement gouvernemental de l'institution asilaire au Québec, loin de s'améliorer avec le temps, tendit dramati-

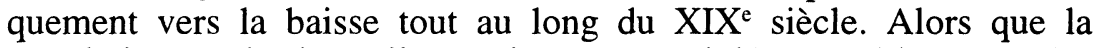
population totale des asiles avait presque triplé entre 1870 et 1885 , passant de 812 à 2272 patients, les subventions gouvernementales consenties pour la même période n'avaient même pas doublé ${ }^{15}$. Lors-

15. En 1870, le budget alloué aux asiles d'aliénés était de $131991,57 \$$. En 1885 , il se chiffrait à $218144,46 \$$. Durant cette période, la loi concernant la participation des parents, des 


\section{GRAPHIQUE 1}

Population des patients publics des asiles d'aliénés au Québec 1850-1912

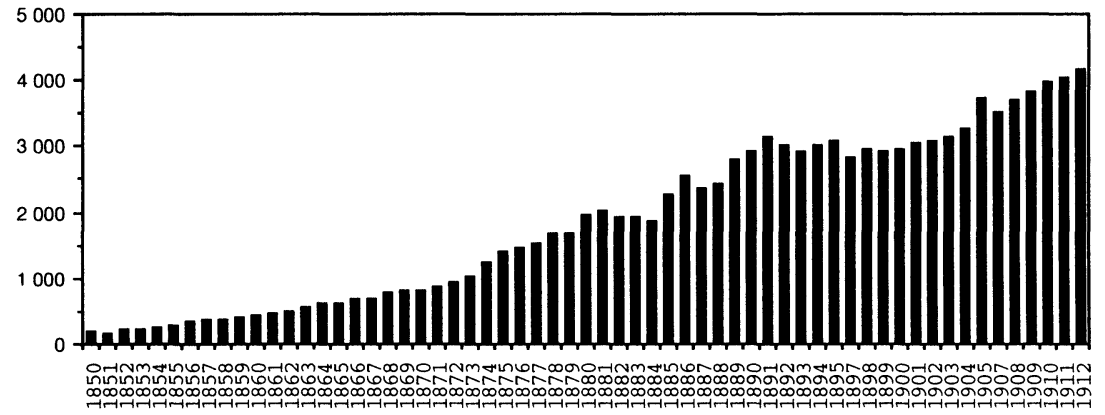

Sources: données tirées des rapports annuels des propriétaires et surintendants d'asiles ainsi que des rapports des inspecteurs d'asiles et de prisons. Le tableau tient compte de l'évolution de la population dans les asiles suivants: Beauport, Saint-Jean-de-Dieu, Saint-Jean d'Iberville, Saint-Ferdinand, Hospice de Baie Saint-Paul, Hospice Saint-Benoit Joseph, Belmont Retreat, Asile protestant de Verdun.

GRAPHIQUE 2

Financement public des asiles d'aliénés au Québec

1871-1912

(en dollars)

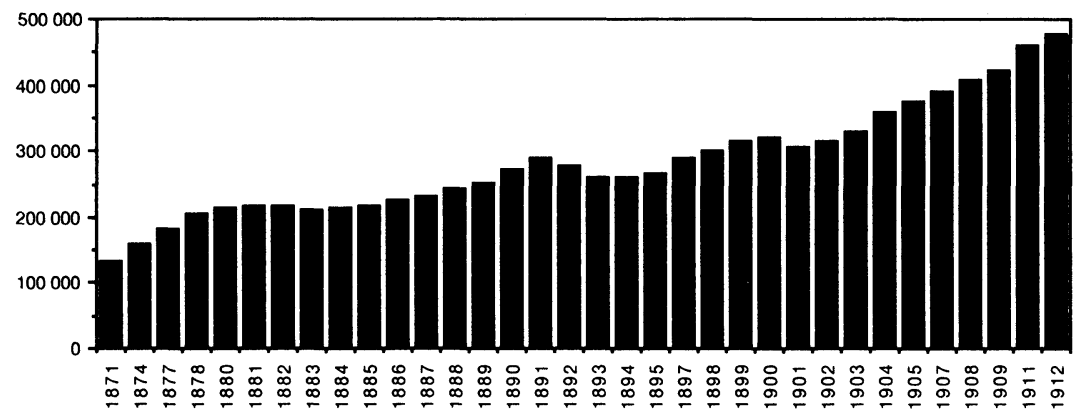

Sources: voir le graphique 1. 
GRAPHIQUE 3

\section{Évolution du financement gouvernemental par patient 1871-1912 (en dollars)}

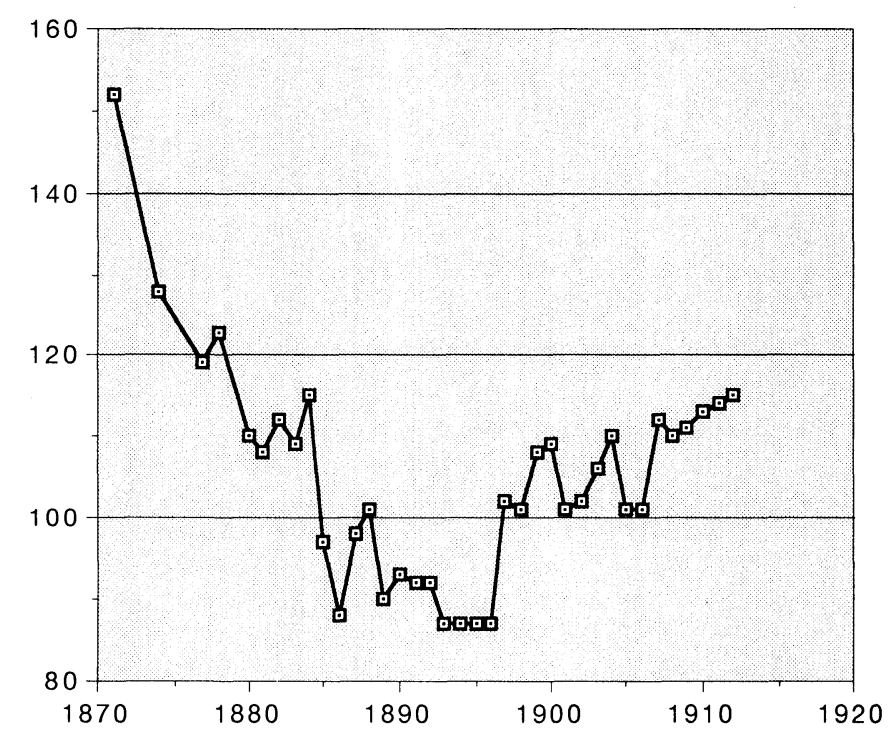

Sources: voir le graphique 1.

qu'on divise le montant global des allocations aux asiles par le nombre total des patients publics subventionnés, on constate que ce financement, qui tournait autour de $152 \$$ depuis 1861 , chuta à $87 \$$ en 1886 et qu'il demeura à ce niveau jusqu'en 1897. Après quoi, il se releva timidement pour atteindre $116 \$$ en 1912.

Cette disparité entre la croissance démographique et l'évolution du financement des asiles est pour le moins surprenante. Sans doute $\mathrm{y}$ a-t-il de nombreuses raisons à cela, mais il y en a au moins deux qui retiennent notre attention. La première, c'est que, bien avant 1870 , le gouvernement avait pris l'habitude de négocier une réduction substantielle pour chacun des patients qui excédait le nombre minimal prévu

amis et des municipalités au paiement des frais d'hébergement dans les asiles n'avait pas commencé à prendre effet. Elle ne sera mise en application qu'en 1884, bien qu'avec beaucoup de difficulté. Le gouvernement comptait alors sur cette loi pour réduire encore plus sa participation financière. 
aux contrats des établissements asilaires ${ }^{16}$. Cette pratique continua par la suite. Concrètement, cela signifiait que plus le nombre de malades hébergés dans les asiles était élevé, moins il en coûtait au gouvernement. Il en allait donc de l'intérêt des pouvoirs publics d'encourager le plus possible la concentration des malades et d'éviter de régionaliser et de multiplier, comme en Ontario, le nombre des établissements. C'était un moyen de limiter considérablement les coûts de construction et d'exploitation. Pour les propriétaires, c'était une invitation à optimiser l'occupation des espaces et à n'entreprendre de nouvelles constructions que là où il n'y avait vraiment plus moyen de faire autrement. Bref, c'était condamner les patients à une promiscuité permanente.

La seconde raison, plus décisive, c'est qu'en confiant le soin d'une bonne fraction de la population des aliénés aux sœurs de la Providence, le gouvernement réalisa ce qu'on pourrait considérer comme une excellente transaction. En effet, en 1870, le montant alloué annuellement aux médecins propriétaires de Beauport pour chaque patient était de $143 \$$ (et de $132 \$$ au-delà de 650 patients), ce qui, incidemment, était déjà bien en-deçà du financement des autres asiles nord-américains ${ }^{17}$; or, à compter de l'ouverture officielle de SaintJean-de-Dieu à la Longue Pointe en 1875, la subvention accordée aux sœurs de la Providence chuta à $100 \$$ par patient et elle ne fut jamais augmentée par après jusqu'à la fin du XIX ${ }^{\mathrm{e}}$ siècle. Dès les premières années d'exercice de Saint-Jean-de-Dieu, la supérieure de l'établissement, sœur Thérèse-de-Jésus, se rendit vite compte de l'insuffisance flagrante de la subvention gouvernementale. Elle revint fréquemment sur cette question dans ses premiers rapports annuels et demanda au chapelain de son établissement, l'abbé Leclerc, d'intervenir en sa faveur. Mais rien n'y fit. Et rien n'empêcha le secrétaire de la province de faire subir le même régime minceur à l'asile de Beauport lorsqu'il passa des mains de ses médecins propriétaires laïques aux mains de la communauté des sœurs de la Charité en 1893. Quant à l'asile SaintJulien de Saint-Ferdinand d'Halifax, qui ouvrit ses portes au début des années 1880 , il n'échappa pas lui non plus aux coupures puisque la communauté des sœurs grises vit le montant de sa subvention annuelle

16. Pour ces patients, avec le contrat de 1873 , le gouvernement ne déboursera que $132 \$$ comparativement à $143 \$$ par tête pour les 650 premiers.

17. Ainsi, par exemple, à l'asile d'État de Halifax, l'octroi annuel du gouvernement se chiffrait à la même époque à 173\$. Mais l'écart était en réalité beaucoup plus grand puisque le coût d'achat et d'entretien de l'asile de Halifax, y compris le salaire du personnel médical et du personnel de soutien, étaient entièrement épongés par le gouvernement. Les propriétaires de Beauport évaluaient le coût global annuel de cet asile à $700 \$$ par patient et ne se gênaient pas pour rappeler au secrétaire de la province la minceur de leur pécule. 
tomber à $80 \$$ par patient ${ }^{18}$. Les raisons de ces dépréciations étaient évidentes: les religieuses, liées par le vœu de pauvreté, représentaient une main-d'œuvre peu coûteuse et surtout peu exigeante. En contrepartie, elles offraient des garanties de fidélité et de fiabilité qu'aucun personnel laïque ne pouvait concurrencer. La loyauté des sœurs offrait l'avantage d'être médiatisée par celle du haut clergé. Et par-dessus tout, la capacité des religieuses, en tant que corporation, d'emprunter et de rembourser le gouvernement avec intérêt pour toute nouvelle construction en faisait certainement un partenaire de choix.

L'asile de Saint-Jean d'Iberville, le seul asile d'État qui ait existé au Québec au XIX ${ }^{\mathrm{e}}$ siècle, ne fut guère mieux financé que les asiles privés: il s'agissait en fait d'un asile temporaire créé à brûle-pourpoint en 1861 pour desservir la région de Montréal et pour répondre à la hausse des demandes d'internement. Cet asile, érigé à même les baraques de Saint-Jean d'Iberville, n'était qu'une une vieille prison maquillée en asile ${ }^{19}$ et sa clientèle était constituée d'une centaine d'exdétenus. Ses bâtiments étaient au bord de la décrépitude et les conditions d'hygiène misérables. Il aurait fallu un investissement considérable pour les rénover, ce qui ne fut jamais envisagé. Cet asile, qu'on se hâta de fermer après une douzaine d'années d'existence, coûta annuellement $285 \$$ en frais d'entretien au gouvernement. Pour un asile d'État, ce budget de fonctionnement aurait été presque acceptable si les bâtiments avaient été convenables. Mais, matériellement parlant, l'asile de Saint-Jean ne pouvait être que préjudiciable pour le médecin qui accepterait d'en assumer la direction. Incidemment, en 1865, les éditorialistes du Canada Medical Journal commentèrent la nomination et la première année d'exercice du docteur Henry Howard en disant: "The treatment received by Dr. Howard [...] whose efficiency has been acknowledged by the inspectors in their last report, has been simply disgraceful, and we would not be at all surprised were that gentleman to relinquish his charge in disgust ${ }^{20} . »$

Seul l'asile protestant de Verdun, ouvert en 1890, fait exception à la règle. Non pas tellement à cause de l'octroi gouvernemental, qui était sensiblement le même que celui en vigueur dans les asiles catholiques, mais à cause de ses structures administratives. Ses promoteurs

18. La raison alléguée était que ces patients étaient des incurables qui n'exigeaient qu'un encadrement minimal.

19. Cf. First Report of the Board of Inspectors of Prisons, Asylums etc. for the Years 1867 \& 1868, Sessional papers, 23 (1869); «The old prison at St-John has been transformed into a temporary asylum for lunatics. [...] The building is moreover so old that it requires unremitting and costly repairs.»

20. Voir «Lunatic Asylums», Canada Medical Journal and Monthly Record of Medical and Surgical Science, 1 (1864-1865): 492. Voir aussi 2 (1865-1866): 331. 
voulurent en effet s'éviter au départ les avatars de la politique à courte vue de l'affermage. Ils renoncèrent délibérément à la formule de la propriété privée, jugée vétuste et contraire aux intérêts des patients et lui substituèrent une formule basée sur la participation financière des milieux aisés anglophones, sur la représentativité et l'électivité administrative, sur la collaboration assidue de la faculté de médecine de McGill et sur la mobilisation générale de la communauté protestante. Ainsi branché sur les ressources du milieu, l'asile de Verdun réussit, malgré son faible pécule de base, à relever considérablement son financement, à se donner l'allure d'un hôpital psychiatrique moderne et à s'attirer une nombreuse clientèle privée. Les avantages de cette formule se réfléchissent dans l'ampleur exceptionnelle des dons et des services consentis annuellement à l'asile, dans la transparence et la technicité de ses rapports annuels, dans les dépenses d'entretien nettement plus élevées pour chaque patient (approximativement le double par rapport aux asiles francophones), dans la libéralité de la discipline et des activités d'animation, dans l'application systématique du traitement moral et dans le rôle nettement prévalent joué par la surintendance médicale. De tous les asiles d'aliénés du Québec au XIX ${ }^{\mathrm{e}}$ siècle, celui de Verdun fut sans aucun doute le premier à concrétiser les ambitions des pères fondateurs de la psychiatrie.

Mais, de façon générale, on peut dire que la politique de financement à contre-courant du gouvernement québécois fut profondément dommageable pour le développement des asiles. Cette politique mettait exclusivement l'accent sur le coût d'hébergement des patients et ignorait presque tout des dépenses nécessaires à l'acquisition d'équipements et au personnel destiné au traitement moral. Ce traitement prévoyait l'organisation sur une base régulière d'activités de loisir et la création d'ateliers de travail pour les patients. Il requérait une maind'œuvre soignante motivée, qualifiée et suffisamment abondante pour impliquer et accompagner les patients dans leurs activités de travail et de loisir. C'est ce qui devait faire de l'asile une institution de cure efficace et sécuritaire, capable de guérir, disait-on à l'époque, près de $90 \%$ des patients, pourvu qu'ils y soient acheminés dès l'apparition de leur maladie. Mais avec leur assiette financière plutôt mince, les propriétaires d'asiles ne purent jamais se payer cette ambition. Le traitement moral ne fut jamais appliqué sérieusement dans les asiles du Québec avant l'arrivée de Burgess à l'asile de Verdun ${ }^{21}$, et c'est plutôt

21. J'ai consacré à Burgess et à l'administration de l'asile de Verdun un article qui devrait bientôt paraitre dans le Canadian Bulletin for the History of Medicine. Burgess naquit à Toronto en 1849. Il fit ses études au Upper Canada College. Diplômé et médaillé (Starr Gold Medal et First University Silver Medal) de la faculté de médecine de l'université de Toronto en 1870 , il entra immédiatement au service de l'asile de cette ville, alors dirigé par le réputé Joseph 
le redoublement des mesures de contention et le confinement permanent des patients à l'intérieur des établissements qui compensa l'insuffisance d'activités et de personnel d'encadrement.

Une autre conséquence du sous-financement étatique fut d'obliger les propriétaires à favoriser des bâtiments de plus en plus volumineux, à étages multiples, ce qui réduisait considérablement le coût des infrastructures et permettait une garde à vue à moindre coût. On prétendait à l'époque que ce système, contrairement à celui des petits pavillons adopté dans beaucoup d'asiles européens, était requis par les particularités du climat. Quoique cela fut vrai d'un point de vue économique (c'était en effet un moyen d'économiser sur le chauffage durant l'hiver), cette politique des gros bâtiments, en plus d'être dangereuse et coûteuse en cas d'incendie, incita davantage au cloisonnement et finit, elle aussi, par contrevenir à l'application du traitement moral. Celui-ci exigeait que les patients puissent circuler le plus librement possible entre l'intérieur et l'extérieur, ce qu'une architecture verticale rendait particulièrement difficile. La politique des gros bâtiments se solda aussi par un véritable cul-de-sac en ce qui concerne la classification rationnelle des patients. La promiscuité et la confusion des diverses catégories de patients, appelés inévitablement à se côtoyer à moins qu'on ne rende étanche chaque étage, engendra un climat d'exaspération qui servit sans doute à merveille les plus délirants, mais fut extrêmement préjudiciable pour les convalescents et les patients en état de rémission.

\section{L'INCENDIE DE 1890 ET LA SATURATION DE L'ESPACE ASILAIRE: L'ASILE COMME PANACÉE HOSPITALIÈRE}

L'adoption de la loi de 1885 , qui transférait la responsabilité du traitement à un Bureau médical, et la création de la Commission royale d'enquête qui s'ensuivit en 1887 ébranlèrent passablement le système d'affermage et eurent des effets positifs sur les propriétaires d'asiles. C'est à ce moment, en effet, que les bourses autant que les idées commencèrent à se délier et qu'on se mit à vouloir moderniser l'asile, à en faire, non plus un hospice, mais un véritable hôpital, au sens moderne du terme. À partir de 1886, la communauté des sœurs de la Providence octroya, en effet, aux docteurs Bourque, Prieur et Barolet, ses médecins traitants, les fonds nécessaires à un voyage

\footnotetext{
Workman, lui-même diplômé de McGill. En 1875, Burgess devint médecin assistant, puis assistant surintendant au London Lunatic Asylum sous la direction de Landor, et, par la suite, sous la direction de Bucke. En 1887, il se retrouva à l'asile de Hamilton où il pratiqua jusqu'en 1890. Il fut le surintendant du Verdun Protestant Hospital for the Insane de 1890 à 1923. Voir American Journal of Insanity, 61 (1904-1905): 163-164.
} 
d'études en Europe. C'était reconnaître que les maladies mentales exigaient comme toutes les autres maladies une évaluation clinique et que les rudiments de connaissances des religieuses ne pouvaient se substituer au savoir d'un professionnel. En 1889, sœur Thérèse-deJésus, la supérieure de Saint-Jean-de-Dieu, entreprit même une tournée des asiles européens et ontariens aux frais du gouvernement. $\mathrm{Ce}$ dernier en profita pour envoyer ses propres médecins visiteurs, les docteurs Vallée et Duquet, au congrès de médecine mentale de Paris.

Ces premiers contacts avec la clinique parisienne et avec les sommités étrangères en psychiatrie débouchèrent sur des initiatives intéressantes: on s'informa plus qu'on ne l'avait jamais fait auparavant des idées de Ball, de Charcot et de Magnan. Faute de professeurs spécialisés dans nos facultés de médecine, on se mit aussi à reproduire dans nos journaux médicaux, à l'instigation des docteurs Laramée et Laberge, de nombreuses cliniques sur les maladies nerveuses et mentales: en l'occurrence celles de Charcot, Pitres, Déjerine, Raymond, Ballet, Debove, Babinski et de la Tourette ${ }^{22}$. On vanta en particulier la qualité de la tenue des asiles anglais et le mérite de certaines expériences tentées en Belgique. On prit note de l'importance de l'enseignement clinique en milieu hospitalier et des mille et une façons d'entretenir le moral aussi bien que la santé physique des aliénés. En principe, on aurait pu croire que le mouvement de médicalisation et de modernisation des asiles s'amorçait enfin rondement au Québec.

Mais, ironie du sort, c'est précisément à ce moment-là que l'asile Saint-Jean-de-Dieu connut la pire catastrophe de son histoire. Le 6 mai 1890, en cours d'avant-midi, un incendie rasa complètement les bâtiments de l'asile ${ }^{23}$. L'incendie fit 86 victimes en plus de disperser aux quatre vents près de 1200 patients. Malgré la construction diligente de pavillons provisoires, cet événement tragique allait reporter

22. À propos des publications parues dans les revues médicales québécoises dans le domaine de la psychiatrie et de la neurologie au tournant du siècle, consulter André Paradis et Hélène Naubert, Recension bibliographique. Les maladies nerveuses et mentales dans les périodiques médicaux québécois (Trois-Rivières, Centre interuniversitaire d'études québécoises, Université du Québec à Trois-Rivières, 1995), 136 p. À ce moment, les recherches cliniques en neurologie et en médecine mentale avaient déjà commencé à progresser dans le milieu médical anglophone de Montréal: George Ross, un chirurgien et un clinicien d'élite, consacrait par exemple des descriptions cliniques et anatomopathologiques détaillées à la chorée, à l'ataxie locomotrice, à la méningite cérébrospinale, à la neurosyphilis, à l'aphasie et à l'hystérie. Armstrong se risquait à opérer dans un cas de tumeur qui donnait lieu à des crises convulsives de type jacksonien tandis que Roddick, qui avait introduit en bonne partie les mesures antiseptiques de Lister au Québec, s'adonnait à la première trépanation dans un cas d'épilepsie (1889). Enfin, James Stewart, qu'on peut considérer comme un des pionniers de la neurologie au Canada, consacra au cours des années 1880 une bonne vingtaine d'articles à la neurosyphilis, au tabès dorsalis, à l'épilepsie, à la méningite cérébrospinale et à diverses formes de paralysie.

23. Consulter à ce propos La Presse, 6, 9, 14, 16, 17 et 21 mai 1890. 
de plusieurs années les espoirs que les médecins francophones avaient commencé à caresser. À partir de 1890, l'encombrement redevint plus que jamais le principal leitmotiv des rapports annuels de la surintendance et la rareté des fonds publics, le principal obstacle à l'organisation rationnelle de la clinique. À l'augmentation ahurissante du nombre des patients, recrutés de plus en plus dans la population francophone et catholique, s'ajoutait l'absence d'hôpitaux spécialisés pour les alcooliques, les épileptiques, les malades chroniques atteints de démence sénile, les aliénés criminels et les déficients mentaux. Rien au budget provincial n'avait été prévu, en effet, pour de tels établissements, et l'institution asilaire, qui ne voyait toujours pas ses revenus augmenter, se retrouvait dans l'obligation de servir de panacée à toute cette gamme d'infortunés.

Durant la période 1895-1910, c'est surtout le docteur Villeneuve qui servit de révélateur au malaise grandissant qui régnait dans les asiles francophones. Villeneuve avait été nommé surintendant médical du gouvernement pour l'asile Saint-Jean-de-Dieu en 1895. Il connut une carrière passablement active autant comme clinicien en psychiatrie que comme professeur et spécialiste en médecine légale. On lui doit de nombreuses initiatives ${ }^{24}$, dont, en particulier, celle d'avoir porté le dossier chargé des asiles sur la place publique.

Dans son rapport pour l'année 1898, Villeneuve fit valoir que l'état d'encombrement était plus que jamais à l'ordre du jour à l'asile

24. Villeneuve demanda au gouvernement une loi plus sévère pour resserrer les critères d'admission des malades âgés, chroniques et à toutes fins utiles irrécupérables. Il plaida avec insistance pour qu'une aide gouvernementale spéciale soit accordée aux épileptiques qui se retrouvaient généralement exclus du marché du travail, démunis, et avec une santé complètement brisée lorsqu'ils arrivaient à l'asile. Il demanda aussi qu'une politique systématique d'inspection des prisons soit élaborée pour dépister les aliénés criminels condamnés ou en détention provisoire qui n'aboutissaient à l'asile qu'après d'interminables tracasseries. Il fit aussi valoir que le traitement des alcooliques était incompatible avec le confinement asilaire, à moins que ces patients ne fussent devenus déments avec le temps. Villeneuve s'impliqua aussi activement dans l'organisation des congrès de 1897 (British Medical Association) et de 1902 (American MedicoPsychological Association), tenus à Montréal, et dans la création de la Société médico-psychologique de Québec (1898), dont il rédigea les règlements en collaboration avec Chagnon. Il en fut aussi le vice-président pour l'année 1899-1900. Villeneuve, comme Chagnon, devint membre de l'American Medico-Psychological Association en 1899 et membre de la Société médicale de Paris l'année suivante. Comme plusieurs autres médecins d'asiles, il participa régulièrement aux congrès annuels de l'association américaine. Il fréquenta à plusieurs reprises $(1884,1890,1894$, 1902 et 1905) les cliniques de la Salpêtrière et de Sainte-Anne et eut comme maîtres Charcot, Magnan, Garnier, Brouardel et Vibert. Il obtint son diplôme d'expert en médecine mentale de la faculté de médecine de Paris en 1895 et, la même année, il accéda au statut de professeur adjoint de médecine légale à la faculté de médecine de l'université Laval à Montréal. En 1897, Villeneuve devint professeur titulaire de clinique des maladies mentales. Cette date est importante si on considère que la clinique asilaire, en milieu francophone, trouva pour la première fois son prolongement dans le curriculum des études universitaires. On doit à Villeneuve près d'une trentaine d'articles sur l'aliénation mentale, la plupart en rapport avec la médecine légale. 
Saint-Jean-de-Dieu et que la situation continuait de se détériorer. Cette situation, selon Villeneuve, était grandement attribuable à «l'affaiblissement du lien de famille et à l'affirmation de l'individualisme» qui faisaient que les classes laborieuses cherchaient de plus en plus, à partir de n'importe quel prétexte, à se débarrasser «de leurs membres inutiles, de ceux qui ne peuvent subvenir à leur entretien ${ }^{25} \gg$. De fait, bien des cas de «démence sénile» ou de paralysie, ni dangereux ni scandaleux, se retrouvaient à l'asile, où on ne pouvait absolument rien pour leur «guérison». Il y avait aussi les nombreux cas d'épilepsie qui n'y étaient pas à leur place. En principe, l'asile était réservé aux malades mentaux curables ${ }^{26}$ et les épileptiques n'y étaient admissibles que si leur maladie s'accompagnait de troubles de déficience majeure ou de délires psychotiques. Mais dans les faits, il en allait bien autrement. Faute d'être bien reçus dans les hôpitaux généraux où ils accaparaient de précieux lits ${ }^{27}$, en plus de semer périodiquement un émoi général, beaucoup d'épileptiques finissaient par «s'incruster» à l'asile après une première admission d'urgence. Cette présence hypothéquait grandement l'espace asilaire. Mais ce n'était pas la seule. D'autres aliénés, des criminels d'habitude aux réactions violentes et imprévisibles, ameutaient aussi périodiquement les autres malades avec qui ils demeuraient en contact. Ils obligeaient à prendre des mesures de sécurité disproportionnées qui risquaient de devenir contagieuses. Il y avait aussi le cas des jeunes déficients que l'asile était forcé d'admettre. Et, comme si cela ne suffisait pas, il fallait ajouter la présence de tuberculeux que, faute de place et de diagnostic méthodique, on ne réussissait pas toujours à isoler en temps opportun. La promiscuité qui en résultait compromettait non seulement les conditions sanitaires et la classification des patients, mais aussi le traitement moral, l'application de la politique de non-contrainte et la cure de repos au lit qui, sous l'influence de Magnan, était devenue le nouveau cheval de bataille de la psychiatrie française ${ }^{28}$. Elle avait aussi un impact direct sur le taux

25. Rapport du surintendant de l'asile Saint-Jean-de-Dieu pour l'année 1898 (Québec, Charles Pageau, 1899), 4-5.

26. Villeneuve rappellera dans son rapport de 1898 la remarque que E.-E. Duquet avait incluse dans son rapport pour l'année 1891: «Les asiles d'aliénés n'ont pas été fondés dans le principe pour l'hospitalisation des vieux ou des vieilles, ni des personnes atteintes de maladies nerveuses. Ils ont pour but de soigner et de guérir les aliénés curables, de protéger les aliénés contre eux-mêmes et d'y enfermer ceux qui sont devenus dangereux pour la société. Ces déments sont une cause d'embarras pour l'asile; ils vicient l'air des quartiers et augmentent le chiffre de la mortalité.» Ibid., 4 .

27. Rapport du surintendant médical de l'hôpital d'aliénés Saint-Jean-de-Dieu pour l'année 1903 (Québec, Charles Pageau, 1904), 11.

28. Rapport du surintendant médical de l'asile Saint-Jean-de-Dieu pour l'année 1900 (Québec, Charles Pageau, 1901), 4 et 8. Consulter aussi à ce propos les rapports du docteur Brochu pour l'asile Saint-Michel Archange, en particulier ceux postérieurs à 1910. 
de mortalité de l'asile: en 1898 , ce taux avait grimpé en effet à 9,43\% à Saint-Jean-de-Dieu et se retrouvait trois fois plus élevé que celui du nouvel asile protestant de Verdun. Dans son rapport pour l'année 1899, Villeneuve résumait la gravité de la situation en écrivant:

La situation actuelle de l'asile de Saint-Jean-de-Dieu, pour employer une expression un peu hardie, est toute dans l'avenir. Elle représente la fin d'une période transitoire qui, par suite des circonstances incontrôlables, s'est prolongée au-delà de toute prévision. Cette époque de transition qui a commencé en 1890, c'està-dire après l'incendie de la maison qui faisait l'orgueil de la révérende soeur Thérèse, a présenté jusqu'en 1897 [...] une incertitude qui a paralysé tous les efforts des révérendes soeurs. Depuis nous sommes forcément entrés dans une période d'attente $[\ldots]^{29}$.

C'était là une façon pour le moins élégante de dire les choses. L'année suivante, Villeneuve décida d'y aller plus franchement: «L'air vicié des salles, écrivit-il, par l'accumulation des malades et la mauvaise ventilation, place les malades dans des conditions hygiéniques tellement défectueuses que l'on peut sans crainte d'erreur attribuer à l'encombrement une bonne proportion de nos décès $[\ldots]^{30} . » \mathrm{La}$ gestion de l'espace asilaire était critique, comme elle l'avait pratiquement toujours été. Et le refus des sœurs de la Providence d'entreprendre la construction d'un pavillon indépendant pour l'administration, comme le souhaitaient les médecins, laissait déjà présager la saturation rapide des nouvelles salles dont l'ouverture était prévue pour le mois d'avril 1901. Les sœurs, qui arguaient l'incapacité financière, étaient loin d'avoir tort. À ce moment, l'investissement du gouvernement de la province dans le secteur asilaire présentait, en effet, une fiche nettement déficitaire. En 1900, malgré un bassin de population comparable, les asiles ontariens hébergeaient 5000 patients comparativement à 3000 au Québec. En outre, chaque établissement ontarien recevait $133 \$$ annuellement «par tête» et les coûts de construction et d'entretien des bâtiments, ainsi que les frais extraordinaires, étaient directement assumés par la province. Au Québec, les religieuses avaient réussi à faire augmenter leur subvention à $114 \$$ par patient, mais elles étaient tenues de payer annuellement, à même ce revenu, une somme de $40000 \$$ pour l'intérêt qui courait sur l'emprunt de un million de dollars qu'elles avaient dû contracter pour la reconstruction

29. Rapport du surintendant médical de l'asile Saint-Jean-de-Dieu pour l'année 1899 (Québec, Charles Pageau, 1900), 4.

30. Rapport du surintendant médical de l'asile Saint-Jean-de-Dieu pour l'année 1900 (Québec, Charles Pageau, 1901), 7. 
de leur asile. Concrètement, cela ne leur laissait qu'une maigre allocation d'environ $85 \$$ par patient pour l'entretien et le développement de leur asile, y compris le traitement du personnel qui était sous leur juridiction $^{31}$. C'était moins que la moitié du montant dépensé annuellement à l'asile de Verdun pour l'entretien de chaque patient! Cette entrée d'argent, quoique «économiquement» exemplaire aux yeux des inspecteurs du gouvernement, était nettement insuffisante. Elle ne permettait pas de répondre à la croissance de la demande et rendait difficile l'application des mesures reconnues comme souhaitables par la profession médicale, à savoir la séparation des cas curables et incurables, la suppression complète de la contrainte mécanique, la généralisation du travail thérapeutique, la multiplication des salles pour la cure de repos au lit et le recrutement de gardiens mieux choisis, mieux rémunérés et donc plus aptes à recevoir une instruction professionnelle $^{32}$. La situation de gêne qui résultait de la rareté des espaces contrecarrait donc l'ambition des médecins de faire de l'asile un véritable hôpital pour les malades mentaux ${ }^{33}$. Chaque décison n'était prise, au dire de Villeneuve, que pour corriger une situation déjà révolue.

Lorsque les nouveaux édifices de Saint-Jean-de-Dieu furent inaugurés en 1904, le docteur Villeneuve en vint à la conclusion que c'était trop peu trop tard et que les conditions de promiscuité faites aux patients et aux membres du personnel continuaient de miner la qualité des soins médicaux. En 1908, la situation était devenue telle qu'une requête dut être adressée directement au lieutenant-gouverneur pour corriger au plus vite la situation financière catastrophique de l'hôpital. Les travaux de construction qui avaient été entrepris cette année-là avaient dû être abandonnés. L'année suivante, en 1909, Villeneuve revint à la charge et signala au gouvernement la difficulté pratiquement insurmontable de mener à bien toute réforme dans son établissement: «À part les inconvénients hygiéniques qu'il présente, dit-il, l'encombrement détruit le principe de la séparation des cas qui est la base du traitement. Cette réforme ne peut se réaliser pour l'hôpital Saint-Jean-de-Dieu que par un agrandissement considérable, tant

31. Ibid., 9-10. Pour une comparaison avec les États-Unis, voir la note 6.

32. Rapport du surintendant médical de l'hôpital d'aliénés Saint-Jean-de-Dieu pour l'année 1903 (Québec, Charles Pageau, 1904), 8-10.

33. Peu de temps après la fondation de la Société médico-psychologique de Québec qui regroupait tous les aliénistes de la province, on envisagea de donner à l'asile Saint-Jean-de-Dieu le nom «mieux approprié d'hôpital». Voir Vingt-huitième rapport des inspecteurs de prisons et d'asiles de la province de Québec pour l'année 1897, Documents de la session, II,9 (18981899): 106. 
pour les besoins présents que pour les besoins futurs. Les efforts individuels menacent de rester infructueux dans la situation actuelle ${ }^{34}$.»

À Beauport, la situation n'était pas tellement plus rose. Dans son rapport pour l'année 1899, Vallée se plaignait, lui aussi, du manque d'espace et de commodités matérielles qui rendait difficilement praticable la politique de mise au travail des aliénés prévue au traitement moral ${ }^{35}$. Il déplorait que les femmes n'aient pas encore de petites chambres et qu'elles soient obligées de passer leurs nuits dans des «cellules» mal aérées et en piteux état ${ }^{36}$. Cette situation, qui tuait dans l'œuf toute velléité de réforme, allait perdurer pendant encore plusieurs années.

Lorsque le docteur Brochu succéda au docteur Vallée en 1903, son intention était pourtant d'appliquer un vaste programme de modernisation. Le grand projet de Brochu était de mettre sur pied une organisation méthodique du travail, de donner plus d'importance au suivi médical, d'améliorer la classification des malades, de faire preuve d'une plus grande libéralité vis-à-vis d'eux et d'adopter des mesures hygiéniques vigoureuses. Ce projet impliquait que les médecins soient délestés de leurs nombreuses tâches administratives ${ }^{37}$ et que les patients chroniques soient séparés physiquement des patients curables. $\mathrm{Au}$ dire de Brochu, les patients en phase de rémission ou en convalescence souffraient beaucoup de l'humiliation de se retrouver confondus avec des cas lourds d'aliénation mentale, de porter, au même titre qu'eux, la livrée obligatoire de l'hôpital et d'être sujets à des mesures de discipline comparables. Il était donc vital de consacrer aux deux grandes catégories de patients, les curables et les incurables, des pavillons différents dans le plan d'agrandissement de Beauport prévu pour l'année 1905. Mais Brochu rencontra vite, lui aussi, l'écueil du financement et dut tempérer ses élans. Les sœurs de la Charité s'opposèrent, en effet, à la construction de pavillons distincts pour les curables et les incurables, manifestement en raison de leurs revenus insuffisants, mais peut-être aussi parce que, dans leur vision des choses, la chapelle devait être au cœur de l'architecture asilaire et facilement accessible à toutes les catégories de patients. Quoi qu'il en soit, en 1909, le personnel de l'asile de Beauport, qui venait d'entrer dans ses nouveaux bâtiments, était encore une fois débordé. Par surcroît, les

34. Rapport annuel du Dr Georges Villeneuve, surintendant médical de l'hôpital d'aliénés Saint-Jean-de-Dieu pour l'année 1908 (Québec, Charles Pageau, 1909), 10.

35. Rapport du surintendant médical de l'asile de Beauport pour l'année 1899 (Québec, Charles Pageau, 1900), 7.

36. Ibid., 9.

37. Rapport du surintendant médical de l'asile de Beauport pour l'année 1903 (Québec, Charles Pageau, 1904), 259-260. 
patients ne pouvaient compter que sur trois médecins, soit un ratio de un médecin par 500 patients. Ce ratio était nettement en-dessous des normes alors en usage en Amérique du Nord.

\section{LES TENTATIVES DE RÉFORME: PRÉVOIR L'AVENIR ET MIEUX DÉLIMITER LA CLIENTĖLE ASILAIRE}

En 1909, au moment où commencèrent à poindre les premières mesures de dépistage et de prévention en hygiène mentale au Québec, Villeneuve demanda des améliorations à la politique d'assistance publique aux aliénés ${ }^{38}$. L'administration asilaire se heurtait, en effet, à deux types de résistance: celle des municipalités et celle des familles. Les municipalités, qui devaient éponger le tiers du coût de l'internement pour chacun de leurs citoyens internés, étaient généralement peu pressées d'intervenir en faveur de leurs malades. Comme chaque municipalité devait voir par ses propres moyens à se faire rembourser par la famille dans les cas où cela était possible, on préférait que les aliénés soient gardés le plus longtemps possible en prison avant leur internement ou encore qu'ils demeurent sous la garde de leurs parents, même si ces derniers étaient inaptes à en prendre soin. D'où la «mobilité» des aliénés physiquement capables qui étaient souvent «encouragés» par leurs proches à «prendre le large». La localisation officielle du domicile de ces «itinérants», de ces délinquants potentiels, donnait alors lieu à des spéculations et à des tracasseries interminables chez les responsables municipaux. Enfin, lors de leur admission à l'asile, la santé mentale et physique de ces «marginaux» était définitivement compromise. Il fallait désormais les compter eux aussi parmi les cas chroniques et incurables.

Par ailleurs, les familles étaient peu portées à faire spontanément des demandes de placement. Seul un petit nombre de patients, capables de payer, étaient admis à l'asile comme patients privés sur demande de la famille. Dans le cas des patients publics, l'internement impliquait non seulement que la famille fasse la preuve de son insolvabilité, mais il fallait aussi qu'il soit clairement établi que le malade à charge était trop dangereux pour lui-même ou pour son entourage, ou encore trop visible du point de vue de la moralité publique. Faute de quoi, les familles devaient soutenir leurs malades jusqu'à la limite du possible, c'est-à-dire jusqu'à ce qu'un événement déclencheur fasse chavirer toute la barque. Il fallait alors bon gré mal gré imaginer une stratégie qui mette les instances publiques dans l'obligation d'agir.

38. Rapport annuel du Dr Georges Villeneuve, surintendant médical de l'hôpital d'aliénés Saint-Jean-de-Dieu pour l'année 1908 (Québec, Charles Pageau, 1909), 10-11. 
Il était donc souhaitable, selon Villeneuve, que le législateur rende obligatoire la déclaration et l'examen médical des malades mentaux confinés à domicile, pour mieux départager les cas, en évaluer la gravité et faire en sorte que, au besoin, ils puissent accéder à l'asile dans des délais raisonnables. Mais ce projet de déclaration obligatoire était difficile à réaliser puisqu'il allait à l'encontre des mœurs et des conventions juridiques concernant le droit à la vie privée. Il fallait aussi, pour accélérer les choses, permettre aux cours de justice d'intenter elles-mêmes des poursuites pour réclamer aux particuliers les montants dont ils pourraient être redevables devant leur municipalité. Cette mesure impliquait logiquement que le législateur comble le vide juridique qui régnait autour de la protection et de la gestion des biens des personnes reconnues comme aliénées et internées dans les asiles ${ }^{39}$. C'était là le moins qu'on puisse revendiquer pour éviter que la situation des asiles, débordés par les cas chroniques, n'aille en se détériorant. L'idéal était et demeurait évidemment que des établissements gouvernementaux distincts soient aménagés pour les épileptiques, les alcooliques, les aliénés criminels dangereux, les chroniques paisibles et les jeunes déficients ${ }^{40}$.

En conviant le gouvernement à cet agenda, Villeneuve ne se faisait pas trop d'illusion, car l'évolution de la clinique psychiatrique ne comptait pas dans les priorités des partis politiques au pouvoir. À partir de 1912, il est vrai, Villeneuve obtint un certain contrôle dans l'admission des patients publics grâce à son ex-collègue, le docteur E. P. Chagnon, qui travaillait alors comme adjoint au Bureau d'assistance municipale de Montréal. Mais comparativement aux asiles nordaméricains, l'asile Saint-Jean-de-Dieu faisait toujours figure de parent pauvre et était encore bien en-deçà du modèle envié des psychopatic hospitals américains. Ces hôpitaux privilégiés, dotés de cliniciens et d'infirmiers spécialisés, se concentraient exclusivement sur les patients aigus et curables et étaient les seuls à offrir des garanties pour le progrès de la clinique des maladies mentales. Villeneuve aurait certainement aimé pouvoir au moins jeter les bases d'un tel modèle. Mais on n'en était pas encore là. En tout état de cause, en 1916, Villeneuve n'avait pas encore réussi à faire bouger les autorités. L'état d'encombrement perdurait dans les asiles du Québec, toujours assiégés par une clientèle tous azimuts. Et la guerre n'allait pas améliorer les choses, car il faudrait désormais ajouter à la faune asilaire tous les

39. Rapport du surintendant médical de l'asile Saint-Jean-de-Dieu pour l'année 1898 (Québec, Charles Pageau, 1899), 7-9.

40. Rapport annuel du Dr Georges Villeneuve, surintendant médical de l'hôpital SaintJean-de-Dieu pour l'année 1915 (Québec, E. E. Cinq-Mars, 1916), 15. 
cas de dépression et d'épisodes psychotiques déclenchés par les déboires financiers, les pertes de conjoint et l'appréhension des événements, et récupérer aussi les ouvriers surmenés des fabriques de munitions de guerre qui tentaient de retrouver leur bonne humeur et leur plaisir dans la consommation élevée d'alcool ${ }^{41}$.

\section{MALGRÉ TOUS LES AVATARS, DES INNOVATIONS MÉDICALES}

Malgré cet état de pénurie et d'engorgement permanents, certains progrès furent enregistrés avant que la Première Guerre mondiale n'éclate. Le plus significatif fut sans doute l'inauguration, en 19021903, d'une chaire de clinique des maladies mentales et nerveuses à l'université Laval à Montréal. Cette chaire fut confiée au docteur Villeneuve qui négocia avec les sœurs de la Providence la possibilité de mettre à la disposition des étudiants les ressources et le vaste matériel clinique de l'hôpital Saint-Jean-de-Dieu ${ }^{42}$. Villeneuve s'adjoignit alors le docteur Joseph-Ernest Dion comme assistant. Dès lors, un petit nombre d'étudiants francophones en médecine purent bénéficier chaque année d'une véritable formation sur le terrain au lieu de simples visites occasionnelles, comme c'était le cas depuis 1888 , c'est-àdire depuis que le docteur Joseph-Edmond Bourque, sur l'insistance du docteur Amédée Lamarche ${ }^{43}$, avait accepté de donner de temps à autre des cliniques improvisées aux étudiants de la faculté de médecine de l'université Laval à Montréal ${ }^{44}$.

À compter de 1903, cette nouvelle chaire devint le lieu de ralliement des spécialistes francophones en psychiatrie. La plupart des cliniciens de Saint-Jean-de-Dieu allaient y passer, soit à titre de démonstrateur, comme J.-Camille Larose, soit à titre d'assistant d'enseignement, comme G. de Bellefeuille et Albert Prévost ${ }^{45}$. La création de cette chaire était d'autant plus opportune que la Société médico-psychologique de Québec, qui réunissait depuis 1898 les aliénistes francophones et anglophones de la province, venait tout juste

41. Rapport annuel du Dr Georges Villeneuve, surintendant médical de l'hôpital SaintJean-de-Dieu pour l'année 1916 (Québec, E. E. Cinq-Mars, 1917), 8.

42. Rapport du surintendant médical de l'hôpital Saint-Jean-de-Dieu pour l'année 1902 (Québec, Charles Pageau, 1903), 7.

43. Le docteur Amédée Lamarche, qui était alors un des éditeurs de L'Union médicale $d u$ Canada, déplorait en effet que «nos écoles [de médecine] ne puissent utiliser davantage un champ si fertile d'observation». Voir Amédée Lamarche, "Visite des étudiants de l'Université Laval à l'asile des aliénés de Saint-Jean-de-Dieu», L'Union médicale du Canada, 17: 111.

44. Une de ces cliniques, consacrée à la paralysie générale, avait d'ailleurs été publiée dans la Gazette médicale de Montréal en 1889.

45. Albert Prévost était médecin légiste, diplômé de l'université de Paris. Dès 1913, il devint professeur agrégé et assistant à la clinique des maladies nerveuses de l'université Laval à Montréal. Prévost était aussi à l'époque neurologiste à l'hôpital Notre-Dame. 
d'être dissoute et que la profession de psychiatre se retrouvait sans aucune plate-forme associative. À ce moment, l'adoption controversée de la loi Roddick (1902) et la multiplication des associations médica-

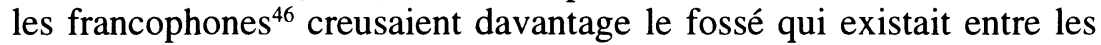
milieux médicaux des deux communautés linguistiques de la province. Cela rendait plus qu'improbable la création d'une nouvelle association médico-psychologique bilingue.

C'est donc à partir de la nouvelle chaire de médecine mentale de l'université Laval à Montréal qu'allait progressivement s'établir un lien organique non seulement entre l'institution asilaire francophone et l'université, mais aussi entre l'hôpital Saint-Jean-de-Dieu et les services médicaux de l'hôpital Notre-Dame ${ }^{47}$. Ce lien commença à se concrétiser en 1911 lorsqu'on finit par admettre qu'un établissement aussi vaste que celui de Saint-Jean-de-Dieu, qui passait le cap des 1200 patients, devait absolument avoir des commodités pour la chirurgie et un laboratoire de pathologie, comme il en existait un à l'asile de Verdun depuis 1898. Ce laboratoire fut donc créé et mis sous la direction du docteur de Bellefeuille qui s'adjoignit, comme assistant, le docteur Larose ${ }^{48}$. Dès la première année d'exercice, Bellefeuille et Larose pratiquèrent plus de 670 analyses dans ce laboratoire (des crachats, du pus, du sang, des sérosités et de l'eau, y compris les tests de Widal, de Eberth et de Wassermann), en plus de s'acquitter de leurs obligations cliniques. C'est peut-être l'obtention anticipée de ce laboratoire qui avait poussé le docteur Alcée Tétrault ${ }^{49}$ à instaurer en 1910 un système de fiches pour enregistrer en détail les données relatives à la santé physique de chaque patient depuis son admission. En octobre 1912, les docteurs Bellefeuille et Larose offraient leurs services aux sœurs hospitalières pour dispenser des cours de clinique répartis sur trois ans $\mathrm{s}^{50}$. Avec son école d'infirmières spécialisées, Saint-Jean-de-

46. Depuis 1885 , plusieurs associations médicales francophones régionales avaient vu le jour. Mais c'est surtout à partir de 1900 que le mouvement associatif francophone va s'intensifier, notamment avec la création de l'Association des médecins de langue française d'Amérique du Nord (1902) et une bonne quinzaine de nouvelles associations médicales régionales. Ce mouvement culminera en 1920 avec la création de l'Association des médecins de langue française du Canada.

47. L'hôpital Notre-Dame servait en quelque sorte d'hôpital universitaire aux étudiants et aux membres de la faculté de médecine de l'université Laval à Montréal.

48. À ce moment, Larose venait tout juste de succéder au docteur S. Robitaille. Rapport annuel du Dr Georges Villeneuve, surintendant médical de l'hôpital Saint-Jean-de-Dieu pour l'année 1911 (Québec, Louis V. Filteau, 1912), 13.

49. Tétrault était le médecin en chef des propriétaires de Saint-Jean-de-Dieu depuis 1906.

50. C'est peut-être à l'occasion de la préparation de ces cours que des médecins d'asiles comme Villeneuve, Tétrault, De Bellefeuille et Larose se remirent à consulter les ouvrages de psychiatrie français dont le fonds Chagnon, de l'université de Montréal, nous a légué une vaste gamme: sous la seule lettre $\mathrm{R}$ du fichier dressé par E.-P. Chagnon lui-même, on retrouve les noms de Régis, Raymond, Rémond, Ribot, Richer, Richet, Roubinovitch, Roux et Roy. 
Dieu troquait définitivement son statut d'hospice pour celui d'hôpital. L'importance prise par l'équipe médicale fut confirmée en 1913 lorsque, en collaboration avec la faculté de médecine de l'université Laval à Montréal, cinq autres services médicaux furent inaugurés et mis à la disposition des patients: l'oto-rhino-laryngologie et l'ophtalmologie (E. Laurent $)^{51}$, l'anatomie pathologique et la bactériologie (G.-Wilfrid Derome $^{52}$, la chirurgie (Eugène Dufresne) ${ }^{53}$, la radiologie (J.-E. Panneton) $)^{54}$ et la neurologie (Albert Prévost) ${ }^{55}$. Presque tous les responsables de ces services étaient déjà rattachés à l'hôpital Notre-Dame. Des salles d'opération furent aussi aménagées avec tout le «matériel aseptique moderne». Au cours de la première année, on y pratiqua 1387 interventions chirurgicales. C'est dire combien il était devenu urgent de compter sur des installations fiables. Finalement, en 1916, un dispensaire des maladies nerveuses et mentales fut créé à l'hôpital NotreDame et mis sous la direction de Villeneuve. C'était une autre façon d'impliquer plus étroitement les étudiants de médecine dans le domaine encore très mal connu de la psychiatrie. Inutile de dire que ces innovations obligèrent le gouvernement de la province à délier les cordons de sa bourse et à hausser son allocation annuelle par patient. Cette allocation passa de 112 à $120 \$$. C'était encore peu. La guerre terminée, d'autres raffinements allaient être apportés à l'arsenal médical de Saint-Jean-de-Dieu, notamment dans le domaine de la dermatologie et de la clinique dentaire.

Parmi les autres initiatives importantes, il faut compter les congés sur essai ${ }^{56}$. La surpopulation asilaire aidant, l'administration médicale des deux asiles francophones de la province décida, en effet, au début des années 1900, qu'il était temps d'accorder à certains patients des «congés d'absence» sur parole. En 1910, 5\% de la population de

51. Laurent fut remplacé à ce poste par E. Foucher en 1915. Foucher était alors l'oculiste et l'oto-rhino-laryngologiste de l'hôpital Notre-Dame et de l'hôpital Saint-Paul.

52. Derome était médecin légiste et chef de laboratoire de l'hôpital Notre-Dame et professeur titulaire de médecine légale à la faculté de médecine de l'université Laval à Montréal.

53. Dufresne était chirurgien à l'hôpital Notre-Dame et à l'hôpital Saint-Paul, professeur agrégé et assistant de clinique chirurgicale à l'hôpital Notre-Dame.

54. Panneton était membre titulaire de la Société d'électrothérapie-radiologie de Paris et directeur du service d'électricité médicale et de radiologie de l'hôpital Notre-Dame. Il était aussi professeur agrégé de radiologie et d'électrothérapie à l'université Laval à Montréal.

55. Prévost, médecin légiste de l'Université de Paris, était assistant à la clinique des maladies nerveuses de l'Université Laval à Montréal et neurologiste à l'hôpital Notre-Dame. Rapport annuel du Dr Georges Villeneuve, surintendant médical de l'hôpital Saint-Jean-de-Dieu pour l'année 1913 (Québec, Charles Pageau, 1914), 3.

56. L'idée des congés sur essai remonte assez loin. Le docteur Howard, qui était surintendant de l'asile Saint-Jean d'Iberville à l'époque, est le premier à y avoir fait allusion dans son rapport publié dans les Documents de la session de $1869, \mathrm{n}^{\circ} 23$. L'idée fut reprise à la fin du $\mathrm{XIX}^{\mathrm{e}}$ siècle par le surintendant du Verdun Protestant Hospital, T. J. W. Burgess. 
Saint-Jean-de-Dieu obtenait déjà un congé. Cette initiative marquait un progrès décisif. Alors qu'auparavant il fallait se comporter dans tous les cas comme si l'asile était un établissement de détention et agir comme si chaque patient était par définition inapte à la vie sociale (l'équivalent du coupable pour l'incarcération), on se mit à relativiser la notion d'internement et à la considérer comme une modalité plus ou moins contingente du traitement médical. Du même coup, on se trouvait à relativiser la notion de guérison puisqu'il n'était plus nécessaire de déclarer un patient guéri pour le rendre à sa famille. On sait à quelles aberrations statistiques avaient conduit au $\mathrm{XIX}^{\mathrm{e}}$ siècle la rivalité entre asiles et la tentation de prétendre officiellement guéris, du seul fait de leur élargissement, des patients dont on savait très bien qu'ils ne l'étaient pas. La nouvelle politique du congé d'absence se trouvait encouragée non seulement par le nombre grandissant de patients privés, internés sur demande de la famille, mais aussi par le désir des médecins de considérer l'asile comme un hôpital, au même titre que tout autre.

Une autre amélioration consista à augmenter le nombre des occupations susceptibles de sortir les patients de leur état de léthargie et de passivité. Alors qu'en 1890 on comptait moins de 15\% de patients impliqués dans des activités de travail, ce pourcentage atteignit respectivement $45 \%$ et $52 \%$ à Beauport et à Saint-Jean-de-Dieu en 1905, comparativement à $60 \%$ pour le Verdun Protestant Hospital for the Insane. Même si ces pourcentages sont vraisemblablement excessifs, c'était une amélioration notoire. L'administration asilaire en retirait un bénéfice certain en termes de décloisonnement et «d'aération», mais aussi des avantages non négligeables en termes de revenus complémentaires. Il y avait cependant un risque à cela, car le nombre de médecins traitants chargés de gérer les activités de travail des patients était beaucoup trop restreint, et le danger de faire de ces activités des occupations purement mécaniques, sans finalité thérapeutique, considêrable ${ }^{57}$.

La fin de la guerre fut marquée par une autre initiative qui consista à organiser des assemblées générales mensuelles pour le personnel médical et des cliniques bihebdomadaires dans les salles de malades. Ce fut au moins le cas à Saint-Jean-de-Dieu. L'esprit de corps et la solidarité entre les médecins des sœurs et ceux du gouvernement s'en trouvèrent renforcés et la concertation sur le traitement et le suivi médical de chaque patient, améliorée. La révision continue des diagnostics et des traitements, les retours critiques sur le travail effectué,

57. C'est implicitement ce que font remarquer les inspecteurs d'asiles et de prisons dans leur rapport de 1909 pour l'établissement de Beauport, 84 . 
la prise en compte des rapports de laboratoire, tout cela fit sentir la nécessité de mieux encadrer et d'impliquer le personnel des gardiens (secteur des hommes) en l'initiant lui aussi aux rudiments de la clinique et du nursing. C'était un des désirs exprimés dans le rapport du nouveau surintendant F. E. Devlin qui venait en 1918 de succéder au regretté Georges Villeneuve.

\section{CONCLUSION}

On comprend peut-être un peu mieux pourquoi il fut difficile pour nos premiers aliénistes psychiatres de faire lever leur discipline avant la fin de la Première Guerre mondiale. Le système de propriété privée des asiles, le financement médiocre de l'institution, la surpopulation chronique, des conditions d'hygiène et de promiscuité peu propices à la santé mentale et physique des patients, les conditions de travail peu reluisantes du personnel laïque, une législation déficiente en ce qui concerne la prévention et le dépistage des aliénés, une vocation beaucoup trop étroite pour l'asile, tout semble avoir joué contre un véritable travail d'exploration des maladies mentales. Avant de pouvoir s'attaquer aux maladies psychiatriques de leurs patients, les médecins d'asiles durent d'abord se battre pour faire de leurs établissements des hôpitaux tout court, c'est-à-dire des lieux hygiéniques, viables et suffisamment équipés pour prendre en charge les maladies physiques et les carences alimentaires qui grevaient presque toujours la santé de leurs patients. Villeneuve, estimait d'ailleurs en 1912 que «le plus grand facteur de guérison des maladies mentales [des] patients venait de l'amélioration des soins matériels et médicaux proprements dits ${ }^{58}$ ». Ce disant, il montrait à quel point la psychiatrie, à l'époque, était d'abord et avant tout dépendante d'une bonne infrastructure médicale et que, sans celle-ci, il était inutile de compter sur les progrès de sa spécialité. Cela était d'autant plus vrai que les psychiatres francophones du Québec se retrouvaient encore, au début du vingtième siècle, dans une situation d'isolement relatif et qu'ils ne pouvaient pas compter, comme leurs pairs américains ou européens, sur le support d'associations professionnelles. Les débats sur la folie n'étaient donc pas très nourris. Il n'est pas sans intérêt de rappeler à ce propos que la très grande majorité des publications des médecins d'asiles québécois pour la période 1890-1920 portèrent non pas sur les maladies mentales, au sens contemporain du terme, mais bel et bien sur les maladies nerveuses, tels la chorée, l'épilepsie, l'alcoolisme ou la neurosyphilis; pour-

58. Quarante-troisième rapport des inspecteurs des asiles d'aliénés, des écoles de réforme et des écoles d'industrie de la province de Québec pour l'année 1912, Documents de la session, 29 (1913-1914): 80. 
tant, compte tenu du continuum qu' on postulait entre les maladies mentales et les maladies nerveuses (d'après la théorie de la dégénérescence de Magnan), on aurait pu s'attendre à ce que la folie morale (les impulsions irrésistibles), tout comme les obsessions paranoïaques, les états hystériques ou la neurasthénie entrent tout autant, sinon plus (vu le matériel clinique existant), dans leurs préoccupations. Si tel ne fut pas le cas, c'est peut-être parce que des pathologies comme l'épilepsie, la chorée ou la neurosyphilis tombaient sans ambiguïté sous la dominance de la neurologie et du modèle biomédical (c'est-à-dire sous la dominance de l'exploration neurophysiologique et anatomoclinique) avec lequel on se sentait, par formation, beaucoup plus à l'aise. Ce ne pouvait être le cas pour les pathologies qu'on qualifiait déjà spéculativement à l'époque de «fonctionnelles». Ces maladies vagues, labiles, sans symptomatologie précise, ces «psychonévroses», comme on avait commencé à les appeler, étaient loin de faire consensus parmi les neurologues. C'est d'ailleurs en postulant que ce «fonctionnalisme» était intrinsèquement inconsistant que Freud, luimême neurologue, allait jeter les fondements de la psychanalyse. 